\title{
Benefits and Constraints of Vitrification Technologies for Cryopreservation of Bovine In Vitro Fertilized Embryos
}

\section{Do $\mathrm{VH}^{1,2}$, Walton $\mathrm{S}^{3}$ and Taylor-Robinson $\mathrm{AW}^{{ }^{* 1}}$}

${ }^{1}$ School of Medical \& Applied Sciences, Central Queensland University, Rockhampton, QLD, Australia ${ }^{2}$ National Key Laboratory of Animal Cell Technology, National Institute of Animal Sciences, Hanoi, Vietnam

${ }^{3}$ Australian Reproductive Technologies, Mt Chalmers, QLD, Australia

${ }^{*}$ Corresponding author: Taylor-Robinson AW, School of Medical \& Applied Sciences, Central Queensland University, Bruce Highway, Rockhampton, QLD 4702, Australia, Tel: 61749232008, E-mail: a.taylorrobinson@cqu.edu.au

Citation: Do VH, Walton S, Taylor-Robinson AW (2014) Benefits and Constraints of Vitrification Technologies for Cryopreservation of Bovine In Vitro Fertilized Embryos. J Vet Sci Anim Husb 2(4): 401. doi: 10.15744/2348-9790.1.505

Received Date: October 22, 2014 Accepted Date: December 10, 2014 Published Date: December 12, 2014

\begin{abstract}
Cryopreservation is the use of ultra-low temperatures to preserve whole living cells and tissues in order to retain their structural integrity and maintain their physiological viability. It enables long term storage of cells in order to circumvent the need for continuous in vitro culture. When cryopreserving bovine embryos there are two means of cryopreservation: slow programmable freezing and vitrification. While controlled-rate and slow freezing can be applied widely to in vivo derived-embryos, this methodology remains less successful for embryos produced in vitro. Vitrification is an alternative technique that minimizes damage due to ice crystal formation and which offers great potential for banking these delicate cells. Examples of circumstances in which this is beneficial include when in vitro fertilization results in more embryos than is necessary for fresh embryo transfer, when conditions are not suitable for immediate embryo transfer, or when transportation of embryos is required prior to use. This paper summarizes the principle of vitrification and addresses its relative strengths and limitations as a means of conserving embryos to facilitate bovine reproductive technologies. Prospective improvements to enhance the efficiency of vitrification and perspectives on its future implementation are also discussed.
\end{abstract}

Keywords: Cryopreservation; Vitrification; Embryo; In vitro fertilization; Bovine; Cattle

\section{Introduction}

Embryo cryopreservation is an important aspect of assisted reproduction technologies [1]. It is feasible to store a number of embryos produced through multiple superovulation and in vitro fertilization (IVF). If conditions are not conducive to bovine embryo transfer, or collection takes place outside the breeding season, then cryopreservation is advantageous. Additionally, exportation of embryos with superior genetics necessitates cryopreservation. It is worth noting that the cryopreservation of embryos and gametes allows us to not only commercialize embryos worldwide, but to establish cryobanks that are indispensable to protection of endangered species and rare breeds.

Regardless of embryo production, all unused embryos are cryopreserved by either controllable freezing methods or vitrification. Slow freezing - using low permeating cyoprotectant concentrations - has been used extensively under commercial conditions [2]. However, while cryopreservation of in vivo-derived embryos is reliable, it does not work well with in vitro-derived embryos $[2,3]$. Therefore, for several reasons vitrification appears to be the cryopreservation method of choice for these embryos. First, the re-expansion and hatching rates of IVF embryos cooled by vitrification are higher than those of embryos frozen by conventional methods [4,5]. Moreover, while conventional cryopreservation is time-consuming [6], vitrification is very simple and cheap [7]. Furthermore, the pregnancy rates of recipients implanted with vitrified IVF embryos were reported recently to be acceptable in commercial settings [8]. The principal reason for this advantage of vitrification is that this technology reduces cryoinjuries to bovine oocytes and embryos [7]; it also minimizes the chilling sensitivity of embryos [6]. Another characteristic of vitrification is that it prevents ice crystal formation during the cooling process by increasing cooling and warming rates [7,8]. Efforts have been made to improve and refine vitrification techniques. Despite promising laboratory results [8-10], vitrification has yet to be employed widely in the field because the majority of embryos are produced from multiple superovulation and conventional cryopreservation is reliable for this source of embryos [2].

While the published literature describes many aspects of embryo cryopreservation to assist bovine reproductive technologies, here we spotlight the principles of vitrification, discuss the advantages and disadvantages of this technology, and consider potential improvements to vitrification and future perspectives on its use. 


\section{Vitrification: high warming and cooling rate method}

Cryopreservation strategies focus on the use of cryprotectants (CPAs) as supplements prior to cooling and the rates of cooling/ warming of the temperature of the sample [11]. Vitrification can eliminate intracellular ice crystals [12], but high concentrations of CPAs are required to achieve the vitreous state [13,14]. CPAs function in a dual capacity by decreasing freezing temperature and increasing viscosity so that instead of crystallizing the syrupy solution becomes an amorphous ice; it 'vitrifies'. While conventional cryoprotectants, such as glycols and dimethyl sulfoxide, are intrinsically toxic to mammalian cells [14,15], Kuwayama [16] has argued that vitrification does not require high concentrations of CPAs such that under special conditions in which the cooling rate is equal to or greater than $107^{\circ} \mathrm{C} / \mathrm{s}[16,17]$, it is possible to induce vitrification with pure water (zero CPAs). Seki et al. [18] also contend that there is an incorrect belief that vitrification is achieved only when cells are exposed to both high CPA levels and rapid cooling rates $\left(>>10,000{ }^{\circ} \mathrm{C} / \mathrm{min}\right)$. However, it is generally accepted that high concentrations of CPAs are necessary to avoid ice formation $[11,18]$. During the freezing process water molecules in biological materials such as cells are removed by the change in the surrounding solute concentration; as the cryoprotectant replaces water, although it is no longer immersed in an aqueous environment the cell retains its native physiological structure and function. Subsequently upon thawing, addition of water during warming reestablishes the original solute concentration [19].

Intracellular ice crystals formed in cooling and warming procedures [18] are the main cause of cell damage [20-22]. During cooling, microscopic ice crystals form that may cause recrystallization in the warming process through a mechanism whereby water molecules transfer from smaller ice crystals to larger ones, which can reach sizes that prove lethal to the surrounding cell [18]. Direct contact of embryos bathed in vitrification solution with liquid nitrogen can increase the rate of cooling [20]. Recent studies have considered the role of warming rates in determining the fate of vitrified cells [18,22]. According to Hopkins et al. [23] differences in warming rates are correlated directly with prior cooling rate because of the accumulation of tiny ice fragments in vitrified cells. A faster cooling rate is required for a corresponding warming rate to block recrystallization [22]. Coupled with moderate cooling rates, high warming rates of $117,500{ }^{\circ} \mathrm{C} / \mathrm{min}$ can protect cells from damage due to recrystallization [18].

\section{Benefits of cryopreservation by vitrification}

It is evident that vitrification has more benefits to offer as a cryopreservation procedure than do slow cooling methods. First, vitrification or ultra-rapid techniques reduce significantly the chilling sensitivity of embryos [6] and prevent cryoinjuries to viable cells from ice crystal formation during the cooling process [2,7,24]. This is due to the use of highly concentrated CPAs [13] and very high cooling and warming rates $[7,8]$. Second, the exposure time to room temperature of cells and tissues is longer for traditional freezing methods than for vitrification [24]. Campos-Chillòn et al. [24] recommend that living cells and tissues should not remain long in such non-physiological conditions before their storage at low temperatures. Third, in regard to costs involved per pregnancy, vitrification is cheaper than stepwise methods [6,24]. Fourth, vitrification is very straightforward [7], not requiring sophisticated machinery, and it is easy for technicians to learn vitrification protocols through a short practical training course [24]. Moreover, in order to evaluate the viability of cryopreserved bovine embryos, in vitro and in vivo procedures are employed. The survival rates of vitrified embryos are often reported higher than those of frozen embryos [13]. Agca et al. [13] also reported that the pregnancy rates of recipients implanted with vitrified embryos are similar to those for whom fresh embryos are implanted. These authors used $0.25 \mathrm{~mL}$ French straws for loading the embryos. However, the sample sizes in their experiments were modest; in the study mentioned above, for example, only 20 embryos were transferred. Similarly, Martinez et al. [25] and Menezo [1] have stated that vitrification is an integral part of IVF programs and can be exploited in commercial conditions.

\section{Constraints of cryopreservation by vitrification}

Although vitrification has several advantages over other cryopreservation procedures it still presents drawbacks. While vitrification minimizes the time exposure of ova and embryos at room temperature [24], this technique may lead to the contamination of embryos during the vitrifying process as a result of direct exposure to liquid nitrogen [7].

Mirabet et al. [26] noted that while liquid nitrogen-mediated soiling may occur on the exterior of containers like straws and bags, it is rare to affect samples contained inside. However, an incorrect assumption is that liquid nitrogen is sterile [27]. Bielansky et al. [28] examined the possibility of viral contamination by introducing into liquid nitrogen three cattle viral pathogens, bovine viral diarrhoea virus (BVDV), bovine herpes-1 (BHV-1) and bovine immunodeficiency virus (BIV). They found that samples in unsealed standard straws tested positive with BVDV and BHV-1 but negative with BIV. In a follow-up study, Bielansky et al. [29] investigated the risk of contamination of sealed plastic straws containing embryos and semen stored for between 6-35 years. Although they detected 32 species of bacteria and one fungus from tested liquid nitrogen, they concluded that there was no risk of contamination for BVDV and BHV-1 in sealed plastic straws and in liquid nitrogen itself. Consistent with this, Mirabet et al. [26] reported the identification of environmental and water-borne bacteria and fungi in liquid nitrogen. It is clear that vitrification is often an open system; therefore, there is a high level of microbial contagion when vitrified samples are banked in liquid nitrogen [27]. Hence, it is necessary to measure the contamination risks in cell and tissue banking. However, it seems that the contamination risks for cryopreserved samples are neglected [11]. 
Despite the fact that vitrification is less time-consuming and is a very simple protocol [6,7], it is necessary to place embryos into vitrification solutions in a timely manner [2]. Technical concerns and those relating to hygiene may be eliminated by handling embryos with care and by using aseptic liquid nitrogen. An acknowledged disadvantage of vitrification is that it is only appropriate for a small number of embryos [2]. In fact, under commercial conditions, cryopreservation of embryos requires a method that allows preservation of a large batch of embryos at the same time [2]. To date, however, this goal has proved difficult to achieve. Consequently, if vitrification is to be used successfully in commercial settings, there are several issues that will need to be resolved.

\section{Improvements to vitrification}

Whenever scientific procedures and methods are established, there is scope to expand upon or enhance those protocols. Many attempts have been made to improve the efficiency of vitrification. Embryologists have sought to either modify vitrification techniques or alter the intrinsic characteristics of bovine oocytes and embryos to make them more cryotolerant to the cooling process.

Modifications to the vitrification process have focused on the type and concentrations of CPAs [16] and on cooling and freezing rates [7]. It is not surprising that in only a few years an abundance of vitrification techniques have been introduced [30]. When van Wagtendonk-de Leeuw et al. [31] investigated the effects of vitrification with a one-step dilution, and slow freezing with a threestep dilution, they found that there was no difference in pregnancy rates for vitrified embryos and slow frozen embryos (45.5\% vs $45.1 \%$, respectively). Notably, however, they showed a significant increase in the survival rates of vitrified embryos after cooling and warming followed by culturing in vitro for 72 hours.

It is well recognized that the formation of ice crystals adversely impacts the survival of embryos and that to minimize these effects the volume of the vitrification solution containing embryos must be relatively small, less than $1 \mu$ [ [32]. Open Pulled Straw (OPS) vitrification - based on a decreased diameter of a $0.25 \mathrm{ml}$ French straw - was developed to meet this demand, where the cooling and warming rates can reach $>20,000{ }^{\circ} \mathrm{C} / \mathrm{min}$ [7]. Similarly, Sanches et al. [8] claim that another vitrification method, Cryotop, can increase the cooling and warming rates up to $40,000{ }^{\circ} \mathrm{C} / \mathrm{min}$. When Morató et al. [33] compared the efficacy of these two procedures for storage of bovine oocytes, they demonstrated that Cryotop vitrification is more efficient than OPS. Interestingly, Matsunari et al. [9] established a conspicuous vitrification method called 'hollow fiber vitrification' that reduces the volume of solution containing embryos to as little as $0.03 \mu \mathrm{l}$. This produced excellent results; the survival rates of murine and porcine embryos after warming and further culturing in vitro were $100 \%$ and $75 \%$, respectively. Furthermore, hollow fiber vitrification also enables the cryopreservation of substantial numbers of embryos. This is especially important for the application of vitrification in field settings. In fact, the findings of Matsunari et al. [9] contradict the retrospective view of Hasler [2], who contends that vitrification is appropriate to preserve only small batches of bovine embryos.

Another approach to improve the efficiency of vitrification is to modify oocytes and embryos in order to make them more cryotolerant. Seidel [34] states that modifications to vitrification procedures often lead to improvements in bovine cryopreservation but acknowledges that limitations remain; therefore, the author asserts that embryos cultured in vitro in a defined medium without supplementation of blood serum can be more cryotolerant than their counterparts cultured in the same medium supplemented with either calf serum or bovine serum albumin. Moreover, there is a correlation between the presence of lipid droplets in embryos and embryo cryotolerance. It is well known that bovine IVF embryos are fatter than same species in vivo embryos [8,35]. Therefore, it is necessary to eliminate the lipid content of bovine oocytes and embryos in order to enhance their cryotolerance to the cooling process. Pryor et al. [10] reported a sophisticated technique to eliminate the fat load of embryos using post-thaw laser-assisted hatching; this method utilizes a laser micro-beam to drill the zona pellucida of embryos to enable lipid droplets contained inside to be released. Sanches et al. [8] also aimed to reduce the lipid content of embryos, in this instance by adding to the in vitro culture medium lipolytic substances, such as forskolin, to reduce intracellular lipids available to cell metabolism. They then used Cryotop vitrification to cool the embryos and impregnated recipients with those treated.

In summary, modifications to vitrification methods that draw upon the intrinsic features of embryos continue to bring about promising outcomes. However, it is important to note that under commercial conditions a novel vitrification method must satisfy the following minimum criteria: (i) achieve good pregnancy rates; (ii) be a method of direct transfer; (iii) be applicable to use in cattle yards since sophisticated facilities are not available on most farms; and hence, (iv) the technique is sufficiently robust to be employed in varied field conditions.

\section{Future perspectives on vitrification}

Worldwide, most bovine embryos are produced by multiple superovulation following exogenous hormone treatment of donor cows. To date, IVF has acquired an increasingly important role in cattle production globally, particularly in North and South American countries [32]. IVF embryo production is both cost-effective and can generate 50 calves per donor per year. It is noticeable that cattle production in vitro can create a plentiful supply of embryos annually, suggesting that numerous surplus embryos may need to be stored for future use. However, as yet, no successful cryopreservation protocol for bovine IVF embryos has been formulated to exploit the large numbers of surplus embryos which, unfortunately, are thus currently often discarded [26]. Hence, TaylorRobinson et al. [32] assert that a novel cryopreservation method is indispensable to the future application of assisted reproductive technologies in large-scale ventures. 
It has been established that traditional freezing reduces to unacceptably low levels upon recovery by warming the viability of bovine embryos in vitro [13]. In contrast, vitrification is considered an effective method to cryopreserve ova and embryos derived in vitro. Many studies [6-8,10,25] have used IVF embryos as a means to test the efficacy of vitrification, and most embryologists now acknowledge that this approach is both practically feasible and highly appropriate. However, the application of vitrification for IVF embryos in the field is limited. The main reason for this is that under commercial conditions, almost all bovine IVF embryos implanted to recipients are fresh rather than frozen. Nonetheless, vitrification provides a promising methodology to facilitate the growing international market for the import and export of IVF bovine embryos. Cryopreservation, and vitrification in particular, is also required to keep track with rapid developments in mammalian reproduction technologies, such as cloning and transgenesis.

\section{Conclusion}

It is apparent that vitrification has advantages over traditional slow freezing methods as a means of cryopreservation of mammalian ova and embryos. The most striking feature of vitrification is that it prevents ice crystal formation during the cooling process [24,32]. Also, this procedure is less time-consuming and relatively cheap compared to conventional freezing protocols [7]. However, the confinement of research on vitrification to the laboratory environment hinders possible opportunities for in situ operation. Although considerable efforts have been made to establish a novel vitrification procedure, large-scale application of IVF bovine embryos remains a relatively unexplored area of research. Evidently, vitrification offers significant potential applications for IVF and cloned bovine embryos in the field.

\section{Acknowledgement}

Financial support for our work is provided by CQUniversity and Australian Reproductive Technologies. We thank the Health Collaborative Research Network for workshop assistance.

\section{References}

1. Menezo Y (2004) Cryopreservation of IVF embryos: which stage? Eur J Obstet Gynecol Reprod Biol 113: S28-32.

2. Hasler JF (2014) Forty years of embryo transfer in cattle: A review focusing on the journal Theriogenology, the growth of the industry in North America, and personal reminisces. Theriogenology 81: 152-69.

3. Dobrinsky JR (2002) Advancements in cryopreservation of domestic animal embryos. Theriogenology 57: 285-302.

4. Mucci N, Aller J, Kaiser GG, Hozbor F, Cabodevila J, et al. (2006) Effect of estrous cow serum during bovine embryo culture on blastocyst development and cryotolerance after slow freezing or vitrification. Theriogenology 65: 1551-62.

5. Nedambale TL, Dinnyes A, Groen W, Dobrinsky JR, Tian XC, et al. (2004) Comparison on in vitro fertilized bovine embryos cultured in KSOM or SOF and cryopreserved by slow freezing or vitrification. Theriogenology 62: 437-49.

6. Mahmoudzadeh AR, Van Soom A, Ysebaert MT, de Kruif A (1994) Comparison of two-step vitrification versus controlled freezing on survival of in vitro produced cattle embryos. Theriogenology 42: 1389-97.

7. Vajta G, Holm P, Kuwayama M, Booth PJ, Jacobsen H, et al. (1998) Open pulled straw (OPS) vitrification: A new way to reduce cryoinjuries of bovine ova and embryos. Mol Reprod Dev 51: 53-8.

8. Sanches BV, Marinho LSR, Filho BDO, Pontes JHF, Basso AC, et al. (2013) Cryosurvival and pregnancy rates after exposure of IVF-derived Bos indicus embryos to forskolin before vitrification. Theriogenology 80: 372-7.

9. Matsunari H, Maehara M, Nakano K, Ikezawa Y, Hagiwara Y, et al. (2012) Hollow fiber vitrification: a novel method for vitrifying multiple embryos in a single device. J Reprod Dev 58: 599-608.

10. Pryor JH, Looney CR, Romo S, Kraemer DC, Long CR (2011) Cryopreservation of in vitro produced bovine embryos: effects of lipid segregation and post-thaw laser assisted hatching. Theriogenology 75: 24-33.

11. Vajta G, Nagy ZP (2006) Are programmable freezers still needed in the embryo laboratory? Review on vitrification. Reprod BioMed Online 12: 779-96.

12. Zhou XL, Al Naib A, Sun DW, Lonergan P (2010) Bovine oocyte vitrification using the Cryotop method: effect of cumulus cells and vitrification protocol on survival and subsequent development. Cryobiology 61: 66-72.

13. Agca Y, Monson RL, Northey DL, Mazni OA, Schaefer DM, et al. (1998) Transfer of fresh and cryopreserved IVP embryos: normal calving, birth weight and gestation lengths. Theriogenology 50: 147-62.

14. Lawson A, Mukherjee IN, Sambanis A (2012) Mathematical modeling of cryoprotectant addition and removal for the cryopreservation of engineered or nature tissues. Cryobiology 64: 1-11.

15. Wu G, Jia B, Mo X, Liu C, Fu X, et al. (2013) Nuclear maturation and embryo development of porcine oocytes vitrified by cryotop: effects of different stages of in vitro maturation. Cryobiology 67: 95-101.

16. Kuwayama M (2007) Highly efficient vitrification for cryopreservation of human oocytes and embryos: the Cryotop method. Theriogenology 67: 73-80.

17. Rall WF (1987) Factors affecting the survival of mouse embryos cryopreservation by vitrification. Cryobiology 24: 387-402.

18. Seki S, Jin B, Mazur P (2014) Extreme rapid warming yields high functional survivals of vitrified 8-cell mouse embryos even when suspended in a half-strength vitrification solution and cooled at moderate rates to $-196^{\circ} \mathrm{C}$. Cryobiology 68: 71-8.

19. Fahy GM (2007) Theoretical considerations for oocyte cryopreservation by freezing. Reprod Biomed Online 14: 709-14.

20. Lee KH, Sun JC, Chuang CK, Guo SF, Tu CF, et al. (2013) An efficient and mass producible method for vitrifying mouse embryos on a paper in cryotubes. Cryobiology 66: 311-7.

21. Mazur P, Pinn IL, Seki S, Kleinhans FW, Edashige K (2005) Effects of hold time after extracellular ice formation on intracellular freezing of mouse oocytes. Cryobiology 51: 235-9.

22. Mazur P, Seki S (2011) Survival of mouse oocytes after being cooled in a vitrification solution to $-196^{\circ} \mathrm{C}$ at $95^{\circ} \mathrm{C}$ to $70,000^{\circ} \mathrm{C} / \mathrm{min}$ and warmed at $610^{\circ}$ to $118,000^{\circ} \mathrm{C} / \mathrm{min}$ : a new paradigm for cryopreservation by vitrification. Cryobiology $62: 1-7$. 
23. Hopkins JB, Badeau R, Warkentin M, Thorne RE (2012) Effect of common cryoprotectants on critical warming rates and ice formation in aqueous solution. Cryobiology 65: 169-78.

24. Campos-Chillòn LF, Walker DJ, de la Torre-Sanchez JF, Seidel GR Jr (2006) In vitro assessment of a direct transfer vitrification procedure for bovine embryos. Theriogenology 65: 1200-14.

25. Martinez AG, de Matos DG, Fumus CC, Brogliatti GM (1998) In vitro evaluation and pregnancy rates after vitrification of in vitro produced bovine embryos. Theriogenology 50: 757-67.

26. Mirabet V, Alvarez M, Solves P, Ocete D, Gimeno C (2012) Use of liquid nitrogen during storage in a cell and tissue bank: contamination risk and effect on the detectability of potential viral contaminants. Cryobiology 64: 121-3.

27. Morris GJ (2005) The origin, ultrastructure, and microbiology of the sediment accumulating in liquid nitrogen storage vessels. Cryobiology 50: 231-8.

28. Bielanski A, Nadin-Davis S, Sapp T, Lutze-Wallace C (2000) Viral contamination of embryos cryopreserved in liquid nitrogen. Cryobiology 40 : 110-6.

29. Bielanski A, Bergeron H, Lau PC, Devenish J (2003) Microbial contamination of embryos and semen during long term banking in liquid nitrogen. Cryobiology 46: 146-52.

30. Kuleshova LL, Lopata A (2002) Vitrification can be more favorable than slow cooling. Fertil Steril 78: 449-54.

31. van Wagtendonk-de Leeuw AM, den Daas JH, Rall WF (1997) Field trial to compare pregnancy rates of bovine embryo cryopreservation methods: vitrification and one-step dilution versus slow freezing and three-step dilution. Theriogenology 48: 1071-84.

32. Taylor-Robinson AW, Walton S, Swain DL, Walsh KB, Vajta G (2014) The potential for modification in cloning and vitrification technology to enhance genetic progress in beef cattle in Northern Australia. Anim Reprod Sci 4: 91-6.

33. Morató R, Izquierdo D, Paramio MT, Mogas T (2008) Cryotops versus open-pulled straws (OPS) as carriers for the cryopreservation of bovine oocytes: effects on spindle and chromosome configuration and embryo development. Cryobiology 57: 137-41.

34. Seidel GR Jr (2006) Modifying oocytes and embryos to improve their cryopreservation. Theriogenology 65: 228-35.

35. Abe H, Yamashita S, Itoh T, Satoh T, Hoshi H (1999) Histochemical and ultrastructural evaluations of cytoplasmic lipid droplets in bovine embryos cultured in serum-free and serum-containing media. Theriogenology 51: 232.

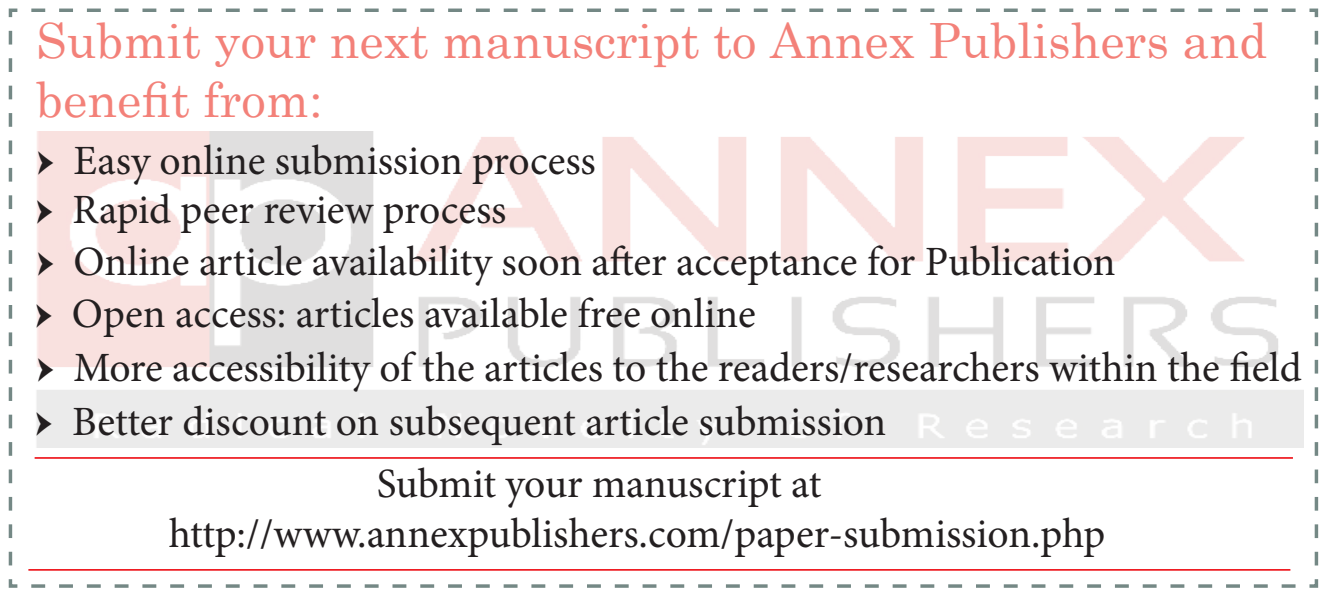

